\title{
Understanding Distributed Systems via Execution Trace Data
}

\author{
Johan Moe \\ Ericsson Radio Systems $A B$ \\ Box 1248 \\ SE-581 12 Linköping, Sweden \\ +46 13-284894 \\ johan.moe@ericsson.com
}

\author{
David A. Carr \\ Institutionen för Systemteknik \\ Luleå Tekniska Universitet \\ SE-971 87 Luleå, Sweden \\ +46920-91965 \\ david@sm.luth.se
}

\begin{abstract}
One of the most challenging problems facing today's software engineer is to understand and modify distributed systems. One reason is that in actual use systems frequently behave differently than the designer intended. We describe a three-step method to allow a developer to understand the run-time behavior of a distributed system. First, remote procedure calls are traced using CORBA interceptors. Next, the trace data is parsed to construct $R P C$ call-return sequences, and summary statistics are generated. Finally, a visualization tool is used to study the statistics and look for anomalous behavior. We are testing this method on a large distributed system (more than 600,000 lines of code) during operation at a customer's site. Despite the fact that the system has been in operation for over three years, we are finding system configuration and efficiency problems using the method.
\end{abstract}

Keywords: CORBA, distributed systems, execution vis ualization, Tracing

\section{Introduction}

Understanding the execution of a distributed software system is one of the most challenging problems facing today's software engineer. During actual use, the system frequently behaves differently than the designer had intended. This may be due to unanticipated use patterns or to a continuously changing runtime environment. Difficulty in comprehension is compounded if the software engineer is not the system's designer - a common occurrence with changing personnel and roles. We are developing a method to improve the understanding and development of distributed systems based on operational data.

Our method consists of three steps:

1. The system's execution is traced during operation at the remote procedure-call level.

2. The trace data is post-processed in order to extract summary statistics and to reconstruct call chains.
3. The post-processed data is studied using information visualization.

While we are currently studying a single large application framework, we feel that the method will be generally applicable to large distributed systems. The current framework, built by Ericsson Radio Systems in Linköping, Sweden, is part of an operation and maintenance $(\mathrm{O} \& \mathrm{M})$ system for cellular telephone networks. The system consists of seven applications and over 600,000 lines of code. Its primary purpose is to provide a toolbox to optimize a radio network. The system has grown from a three-application framework that was originally deployed in 1997.

Our method is in the preliminary stage and currently uses standard tools in all steps. The system uses CORBA to implement its client-server protocol, and our trace mechanism uses CORBA's interceptor definition [19] as the basis for implementation. The raw trace data is then parsed using standard tools (Bison). Finally, the Spotfire.net visualization tool is used to study trace summaries.

Initial explorations have produced promising results. Despite the fact that some portions of the $\mathrm{O} \& \mathrm{M}$ system have been in use for three years, we have discovered design flaws such as, a server raising an exception for a normal case and update requests from user interfaces that were not in use. In addition, a configuration problem was discovered that showed an abnormal number of calls to one of the system services. Performance problems and problems with many iterations over high-level interfaces have also been found.

\subsection{Background}

As a system grows larger, it has a tendency to require more attention from a system's administrator in order to retain performance and quality of service. Currently tools to support understanding of how a system performs during operation are lacking. As a result, system performance 
often degrades during maintenance due to the lack of knowledge about actual product use.

For the $\mathrm{O} \& \mathrm{M}$ system, the problem of operational performance has been neglected - until now. However, more mature customers are now requiring better in-service performance.

Current procedures are inadequate. Most correction and redesign work is based on trouble reports. Nearly all performance and problem data comes from in-house tes ting. These tests focus on function, not performance. Nor, do they attempt to simulate actual use. As a result, what performance information designers receive is usually hearsay from short meetings with customers. This gives a diffuse feeling of performance and is seldom enough information for tuning, rebuilding, or other large decisions.

To solve this problem we need better tools for presenting system performance and system behavior. This should act as help for better manual configuration or $\mathbf{e}$ design. To go one step further, we need tools for automatic configuration of the system, minimizing manual work. The maturity level of an $\mathrm{O} \& \mathrm{M}$ system user varies greatly. Therefore, tools should be more dynamic, fitting different users and their needs at specific moments. In order to build such tools, we need to observe a system as it runs.

Our goal is to build tools that will be used in commercial software development - first, to evaluate and improve current system performance and second, to provide input for monitoring and automatic configuration. In order to insure that our tools solve the system developers' problems, we are working with three different categories of developer: requirement engineers, designers, and testers. Requirement engineers and designers were selected because we believe that they can teach us about tuning and reconfiguration using trace information. Testers were selected in order to learn to find errors using trace information. We also expect to learn something about quality aspects such as test coverage.

\subsection{Building for Use}

For a method or tool to be accepted, it has to be easily integrated into production. From "easily integrated into production", we can state the following requirements:

- The cost for retrieving trace data must be kept at a minimum, i.e., no extra work for designers and low impact on system performance.

- Automatic support for trace data analysis is necessary; manual work should be kept low.

- The visualization must be easy to use, as developers will not spend a lot of time coming up to speed on a new tool. It must also be adaptive to changing developer needs for what data is visualized and how it is presented. Visualization must be flexible, yet focused on its purpose.

The remainder of this paper presents a discussion of our method and preliminary results. This is followed by a survey of related work and finally our conclusions.

\section{Method Description}

Our method consists of three steps: trace, parse, and visualize. We have split it in order to make it more robust and adaptive to change. For example, tracing could be based on another remote procedure call system as long as it provides the same data. Similarly, the parsing can be altered and different statistics generated. In fact, we expect this to happen as we learn more about the operation of the $\mathrm{O} \& \mathrm{M}$ system. Finally, the visualization tool can be changed from Spotfire to a more specialized visualization.

\subsection{Tracing}

Much research has been invested in tracing and tracing applications, as an approach to observing a system. The emphasis has been primarily on debugging during development and in a laboratory setting. Little testing during commercial software development has occurred. Our work explicitly uses trace information during the maintenance phase of a large commercial applic ation.

The first problem is to determine where to add trace points in the system and what to trace. The fact that this was a commercial system imposed the following requirements on the tracing effort:

- No additional work for designers and programmers,

- Have minimal impact on system performance,

- Acquire sufficient data to understand system behavior,

- Don't acquire more data than can be analyzed.

The system we have studied is built on client/server, $n$ tier architecture and uses CORBA. As a part of the CORBA specification, interceptors are defined. Interceptors provide a hook at the remote procedure-call level that, among other things, can help create a tracing mechanism. Using them, we have created a tracing mechanism that captures the client/server communication (e.g. caller, called method, function, parameters, ...). The tracing mechanism can be hooked onto any CORBA server or client during link time $(\mathrm{C}++)$ or runtime (Java). No extra programming is needed. Tracing mechanisms proposed by other researchers often require manual update of source or at best instrumentation, as for example [4]. Such implementation can give great insight from a research perspective. However, the approach is impractical for a commercial development project. 
In order to minimize the impact of tracing on system operations, all data is sent to a remote log server via asynchronous calls. In addition, trace points can be turned on and off dynamically. The protocol between a trace point and the log server is built so that it helps compensate for time differences between hosts. The protocol is also built to reduce network traffic.

In addition to providing a convenient hook, CORBA interceptors provide data at the "right" level to solve performance problems. Since the O \& M system is distributed, performance problems are characterized by the delays required to perform various services. These delays are easily tracked via the call and return behavior of remote procedure calls. Collecting data at this level also reduces the amount of data collected when compared to collecting at the object or module level. We then extract statistics from the trace in order to reduce the amount of data that system designers need to analyze.

Using our design with CORBA interceptors, that send asynchronous messages to a log server, has a couple of other advantages. First, the idea of interceptors allows us to use more than one implementation of CORBA. We have implemented the trace mechanism for two vendors, Orbix from Iona, Inc. and Visibroker from Inprise, Inc. Second, the logging protocol is in itself designed so that we could operate on an RPC mechanism other than CORBA.

\subsection{Parsing}

In the spirit of UNIX filters, three filters are successively applied to raw trace data. First, a filter sorts the raw trace data stream. Then, sequences of interactions are created whenever possible. Finally, statistics are calculated.

The raw trace data is first checked for causality violations and time differences between hosts. This could be called, building a logical event trace from the measured event trace [17]. The method used for time compensation is a tailored version of the algorithm proposed by Hofmann and Hilgers [12].

Next, sequences are automatically built from a number of tracing events. This can be done automatically from information in the raw tracing data. The parser will part $i-$ tion the tracing data into correct and incorrect tracing sequences.

While we can use information directly from previous sessions, we believe that the amount of information is still too high and therefore calculate summary statistics for different time frames, i.e. hour, day, week and month. For the periods, the following is calculated:

- Response time: mean, variance, minimum, and maximum
- Number of calls to a method: completed normally, completed with raised exceptions, and not completed.

- Number of calls between one client (method) and a server (method)

- Amount of data sent

For the moment, the user needs to supply information on what interfaces each server supports. This is used to present functions not called during a trace session (for exa mple, to offer coverage information).

\subsection{Visualization}

We are currently using the "Spotfire.net" visualization tool [22] to analyze the trace summary statistics. "Spotfire" allows one to rapidly construct a scatter plot of the data set in either two or three dimensions. The tool allows specifying and changing the graphical encoding of parameters. If the analyst is interested in the relationship between calls ending in exceptions and those ending normally, these parameters can be chosen as the $\mathrm{x}$ - and $\mathrm{y}$ axes. Other parameters can be configured for presentation such as shape, color, size, etc. "Spotfire" also supports real-time filtering using widgets for specifying the ranges and values. This technique is called "dynamic queries" [1]. The tool also presents a default view based on the order of parameters in the data set.

"Spotfire" is simple to use and allows the analyst to rapidly build a custom visualization. The data display itself does not contain complex visual elements, so learning time is minimal. The power is in the filtering and the real-time response to filter changes. It is simple and quick to set up a custom view to study an aspect of system behavior. An early investigation examined the relative number of exceptions thrown by the system. (See Figure 1.) The axes for calls ending with an exception (y-axis) and calls ending normally (x-axis) are assigned by selecting the parameters from a list attached to the axis. A point with a high number of exceptions was selected, and the "drill-down" details appear in the lower right panel. The details show that the point is for the event server, and all other servers are filtered out using the widgets in the upper right-hand panel.

The final display shows a surprising result for the event server. Most points are above the $45^{\circ}$ line, indicating more than $50 \%$ of all calls ended in exceptions. This indicates a potential problem warranting further investigation. (It turns out that the system was designed to respond to a "no new data" condition by throwing an exception - a questionable practice.)

It should be emphasized that this discovery was a result of exploring the data set. First, the analyst asked what is the relationship between exceptions and normal returns. After constructing a graphic display comparing exceptions and normal returns, a high number of exceptions for 


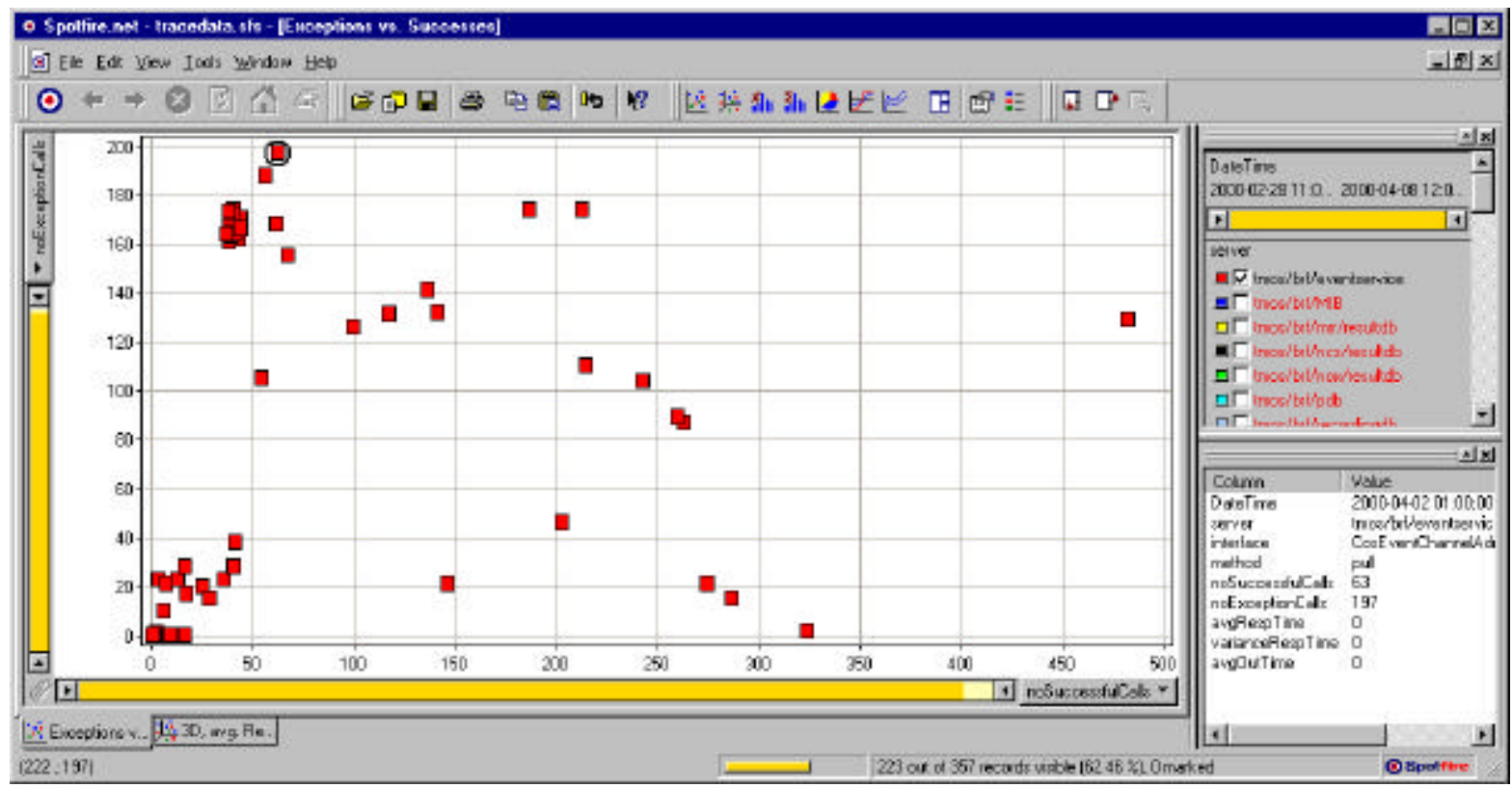

Figure 1. "Spotfire" showing a problem with a high percentage of exceptions. In the leftmost frame the number of successful calls is on the x-axis, and the number of calls ending in an exception is on the $y$-axis. One would expect the rectangles, which represent calls summarized over an hour to be on or near the $x$-axis. However, many show about three calls ending in an exception for every normally terminating call.

the event server was discovered. In order to determine the extent of the anomalous behavior, other servers were filtered out to get a picture of the behavior of the event server. The analyst did not a priori decide to investigate the event server, but rather to investigate the number of exceptions thrown to see if there might be an indication of a problem some where in the system.

Using a standard visualization tool is in keeping with our philosophy of keeping things as simple as possible so that developers don't spend much time learning to use the system. We anticipate that, with time, standardized or customized views may be required. At that time, we will build them.

\section{Preliminary Results}

The method has been used with data from the developer's site during late test activities and from a customer's site during regular system operation. Despite the fact that our system has been in use for only three months, we have had promising results.

Our way of visualizing statistics gives a fast oversight of data. A designer can easily browse the material and get an overview of behavior and performance. One designer said that it was like playing around with the data. This inspired him to look at the data from different views, giving a broader understanding. By selecting different time frames such as hours or days (or perhaps even longer), we seem to find different applications of the method to the development process.

By working with large time frames and collecting information about all possible methods, we have a rough coverage-tool showing all methods not called during a test session. While tracing during system test, it was discovered that several methods were not called. When we investigated this, we received the answer that the trace was during the latter stages of system test and that some tests had been executed before starting the trace. This illustrates that our method provides an efficient way to verify that testing has reached a minimal level of coverage. It is also a good way to insure that regression tests maintain good quality as the system is modified during maintenance.

On the other hand, tracing data from regular operation gave a good view of operational profiles. The customer did not use all functions. As some of the unused functions are one of two alternative ways to accomplish a given function, a discussion started about removing the path that was not used. However, it was decided that data collection at other customer's sites was needed to support such decisions.

By working with small time frames, we have found problems such as unnecessary iteration over interfaces. The drawback is that much of the execution time is used for communication instead of data processing, see Figure 2. Simply put, we identified interfaces and methods with 

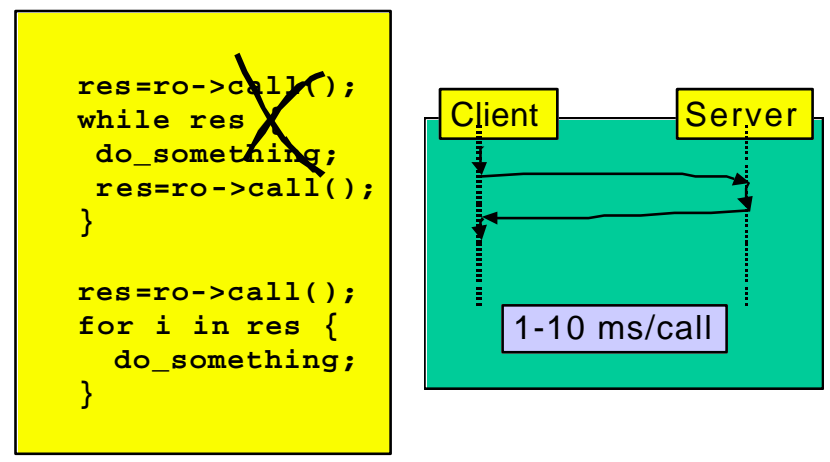

Figure 2. From a performance perspective, we should avoid distributed communication in iterations.

more than one call during a short period of time (one second in this case). Frequent calls indicate that an insufficient amount of information is obtained to justify the overhead of a remote procedure call. Unnecessary iteration over an interface seems to be a common mistake, but it seems to take analyzing a trace of an operational system to catch it. This is an important finding as warning voices within our organization addressed this problem in early 1999. It raises the question as to why the situation has not been dealt with. Our method can partly compensate. With tracing data from actual operations, finding these errors was easy, but not so with data from test execution. The implication is that the system tests need to be revised, and the test data analyzed more carefully, to render realistic system behavior.

During our initial setup, one researcher immediately spotted a strange behavior with one interface. A method had more exceptions than normal returns. This proved to be well known (by some designers) and part of the implementation. The exception was a signal for a polling mechanism to abort operation and run at a later stage. However, it was re-examined and found to consume resources when in fact the system was not in use. This behavior will be removed in a future release of the product.

We also found a server that had not been initialized properly. The server sent messages to another server with a high frequency, every five minutes instead of every 12 hours. A designer immediately reacted to this fact and found the configuration flaw. Finding such a problem in a raw trace would be difficult, as many events will have arrived at the log server during five minutes.

A new version of the $\mathrm{O} \& \mathrm{M}$ system was recently shipped to the customer. During the system test, performance seemed to be satisfactory. However during regular operation, we observed from the trace that a server (the event service) sent events far too frequently. This had the effect that the GUI responded more slowly to user input than expected. For each event, it had to regenerate much of the information, which consumed resources. The same problem could perhaps have been detected by having many users operate at the same time during system test, but observing actual operation is both more realistic and easier.

The more experienced designers found the method promising and tracing at the remote-procedure-call level important. However, when they found a problem, they sometimes wanted finer granularity for debugging purposes. For example, a timestamp should be in microseconds, not as it is now - in seconds. By using the method in day-to-day operations, we hope that feedback from developers will help build a robust and useful system.

\section{Related Work}

Our work draws on work in three related areas: software tracing, sequence identification, and software vis ualization.

Our use of CORBA is not unique. Choi and his colleagues [7] have reported on a tracing mechanism based on CORBA interceptors. Their main concern was to understand the impact tracing had on a system's performance, which they found to be minimal. Similar work has been done by E. S. Cho et al. [6] that showed an approach to clustering CORBA objects from information in a CORBA trace.

Trace approaches can be divided into macroscopic and microscopic [9]. Macroscopic approaches collect cumulative information while microscopic approaches concentrate on sequences of messages. No one approach is better than the other. Both can be used for comprehension, although they focus on different aspects. As an example, we often find macroscopic tools regularly running in an operation environment. With these tools, unwanted behavior can be detected. When a fault is spotted or suspected, 
more detail is usually desired, and a microscopic tool is employed. Our thought is that microscopic tools are necessary and can be of great use in combination with macroscopic tools. For example in our method, tracing sequences are identified. This makes it possible to combine a number of events in a sequence into one logical event. We have used simple pattern matching techniques to extract sequences. Bates [3] has tried a similar approach.

Our method should be seen as macroscopic when it comes to visualization, and is similar to the objectoriented macroscopic visualization of R. J. Walker, et. al. [23] and W. De Pauw et. al. [8]. However, we are not focused on object-oriented systems, but on large distributed systems instead. We have found that as tracing sessions grow longer, one can perceive the stream of tracing data as infinite. Therefore, we present information summary statistics by time period (per hour, etc.). This is in order to build detailed operational profiles [18].

One uses microscopic approaches to learn how something is accomplished or to get more detail. Examples of this are Karam [14] and De Pauw, et. al. 9]. Karam shows execution traces on a timeline where it possible to zoom in and out. His system provides a state-machine based interpreter for events. This, in turn, is used to construct timelines using an oscilloscope metaphor. The metaphor is further enhanced with annotation symbols that overlay the "signal trace". De Pauw's work focuses on displaying execution traces in an object-oriented system. They use Jacobson's interactive diagram [13] as a basis for visualizing execution sequences. They introduce a modification to the diagram to make recursion explicit and introduce several methods to compress the diagram. Two such methods, called "flattening" and "underlaying", are shown in Figure 3.

Frumkin and his colleagues report on a system that is aimed at debugging of message-passing parallel programs that also includes the possibility of replaying execution

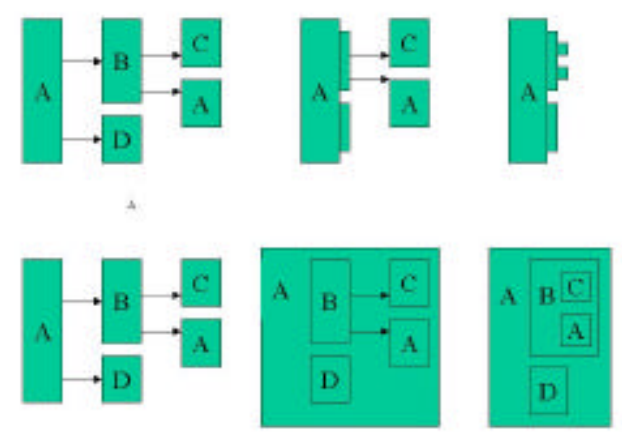

Figure 3. The top relationship is "flattened" by collapsing intermediate events. The bottom relationship is "underlain" with containment replacing directed arcs.
[11]. They augmented a communication library and provide two versions, with and without tracing. Their tracing must be enabled at link time by selecting the appropriate version, which is not as flexible as the run-time enabling provided by CORBA interceptors.

Renieris and Reiss 20] have taken a different approach, and automatically insert tracing calls into the program. They provide an active display with the ability to jump from the displayed tracing data to the source code. In addition, they use a spiral to depict trace data. This makes better use of screen real estate by providing a denser image than linear methods.

Automatic fault detection has been tried by a number of researchers. For microscopic approaches, automatic loop detection has been tried in De Pauw, et. al. [9] and J. R. Larus [16]. A totally different approach to fault detection is the use of query languages. Here, the user simply asks questions about the tracing data. The problem is, that one needs a good idea of what one desires. This is often not the case, especially for maintenance programmers who are unfamiliar with the system. A good example of a query language is PARFORMAN [2]. With this language the authors demonstrate approaches to race detection, deadlock, and other concurrent programming problems.

Software visualization has traditionally concentrated on the source code as a basis for the visualization. Various metaphors have been used in order to increase understanding. SeeSoft [10] used a source-file-based metaphor with files represented by boxes and with source code lines represented by colored lines within the boxes. The emphasis was on displaying various aspects relating sourcecode-modification history, although SeeSoft also provided a test-coverage visualization. The call graph and class diagram have also been used as a basis for software visualization. Recent examples include C++ Lens [5], Software World [15], plus Schauer and Keller's patternbased tool [21]. The C++ Lens displays inheritance hierarchies as trees and uses size encoding to emphasize relationships such as the number of references. Software World uses a cities-neighborhoods-buildings metaphor to represent software inheritance and containment relationships. Schauer and Keller's tool uses a network to display interclass relationships with design patterns as an organizing theme.

Source-code-based visualizations primarily emphasize static aspects of software. Walker, et. al. [23] report on a trace visualization system that concentrates on dynamic aspects of object-oriented systems. Their system uses animation to convey dynamic relationships, and boxes and arrows to convey static relationships.

None of the above systems provide for real-time dynamic filtering that can be obtained using dynamic queries [1]. Some, such as SeeSoft, allow for user customization of their display. 


\section{Conclusions and Future Work}

It truly seems that most designers do not completely understand a large system's behavior. This holds for many aspects such as call frequencies. For example in the $\mathrm{O} \& \mathrm{M}$ system, there is a daemon for handling and serving specific event notifications to one of the servers. The person responsible for implementation said that there should be something like 200 calls for a specific situation. In fact, tracing showed actual rates were one order of magnitude greater. In order to provide better information, we have collected operational trace data from a system in regular commercial use. This data has been parsed and then displayed using information visualization techniques so designers may obtain a better understanding of the system's actual behavior.

With an early version of our method, we can see improvement in designer's understanding of their system's operational behavior. The method has been used to discover problems in a system that has been in operation for over three years. These problems include using an exception to signal a normal condition, iterative calls to an interface due to inadequate buffer size, and inadequate test coverage. Correcting some of these problems has improved system performance. Typically, these errors can be difficult to express in a trouble report. Therefore without methods like the one proposed, problems remain and annoy users for some time.

We have now started to see patterns of common errors in particular repetitively calling an interface because a buffer has been inadequately sized. The method should be able help both designers in correcting these faults and organizations in preventing common problems. For example, a line manager can start specific educational $\mathfrak{x}$ tivities to prevent common mistakes.

Although we have had early successes, much still needs to be done. One thing that we see is the need for more flexibility with regard to time scale. It seems clear that we need the ability to study data at both large and small time scales. The best time window depends on one's current goal. It also seems that we need more flexibility in working with sequences as well. In addition to calculating statistics, we would like to examine execution details when statistics signal anomalies.

With macroscopic collection and presentation, more than just designers can take advantage of the trace information. For example, information can be used for $\mathbf{e}$ moving unused functions or prioritization during maintenance. Also, testers can use the information to improve test reality and begin to test issues such as performance in addition to functionality.

From a staff-development point of view, we see that additional integration with source code and documentation is necessary. For example, new developers might use the visualization to learn what the most commonly exe- cuted services are. A display showing frequency of execution versus time might be used. After selecting a point near the top of the scatter plot, developers would obtain the server and method names using the drill-down feature. At this point, they would need to leave the visualization in order to obtain further information. However, providing a link from the details obtained by drilling-down to related documentation and source code would be better.

We also see that the information gained in studying the system could be used to construct automatic monitoring tools for the operations staff. The trace could be monitored and compared against "normal" behavior. With a realistic model of system behavior, this information might be used to signal potential problems as they developed. This could lead to dynamic tuning to prevent or mitigate them. The data might also be used to plan expansion and upgrades.

In summary, we are confident that by tracing distributed systems at the remote procedure-call level we can gain insight and understanding about how the system operates, while paying a low price. This knowledge can be used to improve the system throughout the maintenance phase of its life.

\section{Acknowledgments}

This research has been in part funded by the Stiftelsen för Kunskaps- och Kompetensutveckling through their Industrial Graduate School Program. We would like to thank Kristian Sandahl for constructive comments. Fnally, we would like to thank Carl Rollo for proofreading.

\section{References}

[1] Ahlberg, C., Shneiderman, B. (1994) Visual information seeking: tight coupling of dynamic query filters with starfield displays. Proceedings of CHI'94, ACM Conference on Human Factors in Computing Systems, Boston, MA, April 24-28, 313-317.

[2] Auguston, M., Fritzson, P. (1996) Parforman - an assertion language for specifying behaviour when debugging parallel applications. International Journal of Software Engineering and Knowledge Engineering, 6(4), 609-640.

[3] Bates, P. C. (1995) Debugging heterogeneous distributed systems using event-based models of behaviour, ACM Transactions on Computer Systems, 13(1), 1-31.

[4] Bruegge, B., Gottschalk, T., Luo, B. (1993) A framework for dynamic program analysers, OOPSLA'93, ACM Press, Washington, DC, Sep 1993, 65-82. 
[5] Cain, J. W., McCrindle, R. J. (1999) Software vis ualisation using $\mathrm{C}++$ lenses. Proceedings of the 7 th International Workshop on Program Comprehension, Pittsburgh, PA, May 5-7.

[6] Cho, E. S., Kim, C. J., Kim, S. D., Rhew S. Y. (1998) Static and dynamic metrics for effective object clustering. Proceedings of the Asia-Pacific Software Engineering Conference (APSEC '98), Taipei, Taiwan, Dec 1-4, 78-85.

[7] Choi, C.H., Choi, M.G., Kim, S.D. (1998) CSMonitor: a visual client/server monitor for CORBA-based distributed applications. Proceedings of the Asia-Pacific Software Engineering Conference (APSEC '98), Taipei , Taiwan, Dec 1-4, 338345.

[8] De Pauw, W., Kimelman, D., Vlissides, J. (1994) Modeling object-oriented program execution. Proceedings of the $8^{\text {th }}$ European Conference on ObjectOriented Programming (ECOOP '94), Bologna, Italy, July 4-8, 163-182.

[9] De Pauw, W., Lorenz, D., Vlissides, J., Wegman, M. (1998) Execution patterns in object-oriented visualisation, Proceedings of the Fourth USENIX Conference on Object-Oriented Technologies and Systems, Santa Fe, NM, April 27-30, 219-234.

[10] Eick, S. G., Steffen, J. L., Sumner, E. E. (1992) SeeSoft-A tool for visualising line oriented software statistics. IEEE Transactions on Software Engineering, 18(11), 957-968.

[11] Frumkin, M., Hood, R., Lopez, R. (1998) Tracedriven debugging of message passing programs, Proceedings of IPPS/SPDP'98, Orlando, FL, Mar 30 - Apr 3, 753-762.

[12] Hofmann, R., Hilgers, U. (1998) Theory and tool for estimating global time in parallel and distributed systems. Proceedings of the 6th Euromicro Workshop on Parallel and Distributed Processing, $P D P^{\prime} 98$, IEEE Computer Society, Madrid, Spain, Jan 21-23.
[13] Jacobson, I., Christerson, M., Jonsson, P., Övergaard, G. (1992) Object-Oriented Software Engineering: A Use Case Driven Approach, AddisonWesley, ISBN 0-201-54435-0.

[14] Karam, G. M. (1994) Visualising using timelines. Proceedings of the 1994 International Symposium on Software Testing and Analysis, Seattle, WA, Aug 17-19, 125-137.

[15] Knight, C., Munro, M. (1999) Comprehension with[in] virtual environment visualisations. Proceedings of the 7th International Workshop on Program Comprehension, Pittsburgh, PA, May 5-7.

[16] Larus, J. R. (1999) Whole Program Paths, Proceedings of the ACM SIGPLAN '99 Conference on Programming Language Design and Implementation, Atlanta, GA, May 1-4, 259-269.

[17] Malony A. D. (1991) Event-based performance perturbation: a case study. Proceedings of the Third ACM SIGPLAN Symposium on Principles Practice of Parallel Programming, Williamsburg, VA, Apr 21-24, 201-212.

[18] Musa, J. D., (1993) Operational profiles in software reliability engineering, IEEE Software, 10(2), pp. 14-32.

[19] Object Management Group (1998) The common object request broker: architecture and specification. revision 2.2, Framingham, MA, USA.

[20] Renieris M., Reiss, S. P. (1999) Almost: exploring program traces, NPIVM' 99 Workshop, Kansas City, MO, Nov 6.

[21] Schauer, R., Keller, R. (1998) Pattern visualisation for software comprehension. Proceedings of the 6th International Workshop on Program Comprehension, Ischia, Italy, June 24-26.

[22] Spotfire, A. B., http://www.spotfire.com/.

[23] Walker, R. J., Murphy, G. C., Freeman-Benson, B., Wright, D., Swanson, D., Isaak, J. (1998) Visualizing dynamic software system information through high-level models, OOPSLA'98, Vancouver, Canada, Oct 18-22, 271-283. 